\title{
EDITORIAL
}

\section{Editorial: a new editor takes over}

\author{
European Journal of Clinical Nutrition (2012) 66, 1-2; \\ doi:10.1038/ejcn.2011.185
}

Taking on the role of Editor of EJCN is a challenging prospect. EJCN has a long tradition and a long list of outstanding scientists who have held this position before me. The list began with John Waterlaw and now ends with Prakash Shetty, from whom I have taken over. Each of the previous Editors have tried to improve and maintain high standards-as Prakash Shetty mentioned in his last editorial (Shetty, 2011), all strived to 'promote the growth and success' of EJCN. I would like to take this opportunity to congratulate Prakash and thank him for his excellent services to the journal, while I remember fondly the time I spent recently in a pub in London with him.

Today the merit of a scientific journal is mainly, if not all, about the impact factor (IF); it is this that seems to be the predominant influence when authors are confronted with a vast array of titles to choose from for the publication of their research. Faced with the present IF, a new Editor may ask whether he has the mandate to proceed to improve the journal and further increase its IF? Difficult to say and difficult to reach. To begin with we have to think about the 'nature' of IFs, what we currently do in an effort to increase IF and what we will go on to do to further our efforts in the future. Key to this part of the job is alluring papers, which besides their scientific value may have the potential to reach a high citation rate, and thus, may significantly add to the journal's IF.

Although clearly important, continuing to attract high quality articles is just a one option to consider when thinking of how to develop a journal? I would like to begin my job as the Editor of EJCN by addressing four other areas that I feel are important-scope, structure, human touch and, what I would like to call, the 'outfit' of the journal.

Scope: Nutrition including clinical nutrition is applied physiology together with applied biochemistry. Our discipline delivers advances in understanding of metabolism and its regulation as well as diagnosing and treating inborn or acquired diseases related to nutrition. Within the last 30 years we were and are still faced with what has been frequently called a genomic or molecular 'revolution'. As in other biomedical sciences, this has shifted the balance between physiology and molecular biology in the science of nutrition. This is reflected by the names of new departments and also new journals on molecular nutrition. These developments have also favoured some kind of reductionism leading to a detailed and in-depth view of mechanisms. By contrast genomics and molecular nutrition did not (as I feel) add much to a greater understanding of nutritional problems or to better treatment of our patients. As, however, the fundamental issues of clinical nutrition remain the same and are still challenging in daily practice, there is some disappointment and also some frustration within our scientific community. Some colleagues consider this as a relative failure of the molecular 'revolution' and consequently the enthusiasm for gene-centred medicine is vanishing. To be positive, I feel that we will come back together. System biology and so-called 'integrative physiology' provide a basis for future and fruitful cooperations. $E J C N$ is open for both areas of research, obviously, there is need for integration. Our journal will provide a suitable forum of what may be called 'integrative nutritional science'. And although EJCN is named a European journal, we are open to the entire scientific community all over the world.

Structure: A good journal has to be readable. This depends in part on its structure. Going through the contents of each of the past issues of EJCN, it is evident that there is need for structuring at least according to the different areas of nutrition research. This adds to focus and will also bring together corresponding articles (for example, on hot topics). It may also add to stimulate discussion. Ideally, a clear structure will stimulate authors to contribute to our discussion and to submit their respective manuscripts.

Human touch: It is a matter of fact that the work of the Editorial Board members greatly contributes to the quality of a scientific journal. But do we know who they are, what they think or even how they look? EJCN has a highly reputed international Editorial Board covering the whole spectrum of research in all areas of clinical nutrition. In future issues, we will introduce each of our Editorial Board members one-byone via a 'Getting to know...' questionnaire, in order to bring them nearer to the readers and authors. 'Getting to know...' will not only be about residence, career, position and major research interests, but will give the reader an insight into their thinking and their personality. After presenting all the members you will have a complete picture of our Editorial Board, hopefully including visions and orientations, adding to what I call the 'internal' culture of EJCN.

'Outfit': A good journal should also be enjoyable. Ideally we all-the readers, authors, Editors, as well as the members of staff from the Nature Publishing Group-should like and want to read the journal. Initial attraction has an important 
role to play in drawing readers to the journal, despite the well-known saying 'Don't judge a book by its cover'. Going back through the past issues of EJCN the front cover has remained the same for some time now. Clearly, EJCN should not become a popular magazine but something should be done to improve attractiveness and outfit. Thus, starting with the January 2012 issue, each issue of EJCN will have a photo on its front cover. This should be an eye catcher-an image that will catch the attention of readers and that they won't forget. Maybe a scientific journal can also raise some emotions related to liking and wanting (I am not a psychologist but I liked to hear John Blundell speaking about that issue). If so, the new Editor feels that that would be ok.

I put these ideas to you with an open mind. Maybe you will agree with them. Maybe you will consider them as too much or going into the wrong direction. Maybe you like $E J C N$ as it is. Maybe you have alternative ideas, which I would be very pleased to receive. Each idea has its right and is welcome. I am sure that all of our readers will agree that a new Editor should have a chance. Within the next months we will see how my initial ideas will work.

Just a last word, or perhaps a personal note. My parents taught me that Germans should always be reserved. This was and still is because of our recent history. Although many years and nearly three generations have passed by, I still feel that my parents were right. Becoming the Editor of EJCN is something like loosing that reservation and taking over. As a German it is thus mandatory for me to handle the position very carefully. Each of us has a self-concept, which is, as one sooner or later admits, sometimes better than the real life experience. I have a global perspective, am positive and open minded. As such I would like to invite researchers and colleagues from inside and outside Europe and North America to submit their contributions to EJCN. I hope that most, if not all, of you will accept my invitation. If so that would be a great experience, which goes far beyond the IF. As the new Editor of EJCN, I am looking forward to an exciting, challenging and fruitful time.

\section{Conflict of interest}

The author declares no conflict of interest.

MJ Müller

Universität Kiel, Institut für Humanernährung und Lebensmittelkunde, Kiel, Germany E-mail:mmueller@nutrfoodsc.uni-kiel.de

\section{Reference}

Shetty P (2011). Eur J Clin Nutr 65, 1-2. 